(2) OPEN ACCESS

\title{
Parental psychological distress during pregnancy and the risk of childhood lower lung function and asthma: a population-based prospective cohort study
}

\author{
Evelien R van Meel (1) 1,2 Gautam Saharan, 1,2 Vincent WV Jaddoe, 1,3 \\ Johan C de Jongste, ${ }^{2}$ Irwin KM Reiss, ${ }^{4}$ Henning Tiemeier, ${ }^{5,6,7}$ Hanan El Marroun, ${ }^{3,5,8}$ \\ Liesbeth Duijts (i) ${ }^{2,4}$
}

For numbered affiliations see end of article.

\section{Correspondence to}

Dr Liesbeth Duijts,

Epidemiology, Erasmus Medical Center, Rotterdam 3000, The Netherlands;

I.duiits@erasmusmc.n

ERvM and GS contributed equally.

Received 18 September 2019 Revised 10 August 2020 Accepted 13 August 2020 Published Online First 12 October 2020
Check for updates

(C) Author(s) (or their employer(s)) 2020. Re-use permitted under CC BY. Published by BMJ.

To cite: van Meel ER, Saharan G, Jaddoe VWV, et al. Thorax 2020;75:1074-1081.

\section{ABSTRACT}

Background Although maternal psychological distress during pregnancy is associated with increased risks of respiratory morbidity in preschool children, it is unknown whether this association persists into later childhood. Objective To examine the association between parental psychological distress during pregnancy and lung function and asthma in children of school age. Methods This study of 4231 children was embedded in a population-based prospective cohort. Parental psychological distress was assessed by the Brief Symptom Inventory during and 3 years after pregnancy, and in mothers also at 2 and 6 months after pregnancy. At age 10 years, lung function was obtained by spirometry and asthma by questionnaire.

Results The prevalence of asthma was 5.9\%. Maternal overall psychological distress during pregnancy was associated with a lower forced vital capacity (FVC) (z-score difference -0.10 (95\% Cl -0.20 to -0.01$)$ per 1 -unit increase), maternal depressive symptoms during pregnancy with a lower forced expiratory volume in the first second $\left(\mathrm{FEV}_{1}\right)$ and $\mathrm{FVC}(-0.13(95 \% \mathrm{Cl}-0.24$ to -0.01$)$ and $-0.13(95 \% \mathrm{Cl}-0.24$ to -0.02$)$ when using clinical cut-offs) in their children. All maternal psychological distress measures during pregnancy were associated with an increased risk of asthma (range OR: $1.46(95 \% \mathrm{Cl} 1.12$ to 1.90$)$ to $1.91(95 \% \mathrm{Cl} 1.26$ to 2.91)). Additional adjustment for paternal psychological distress during pregnancy and parental psychological distress after pregnancy did not materially change the associations. Paternal psychological distress during pregnancy was not associated with childhood respiratory morbidity.

Conclusion Maternal, but not paternal, psychological distress during pregnancy is associated with an increased risk of asthma and partly lower lung function in children. This suggests intrauterine programming for the risk of later-life respiratory disease.

\section{INTRODUCTION}

Early life is a sensitive period for the development of respiratory health. ${ }^{1}$ We and others have previously shown that maternal psychological distress during pregnancy is associated with increased risks of wheezing and asthma in their preschool-aged children. ${ }^{2-7}$ This suggests a potential role of intrauterine mechanisms, such as altered programming

\section{Key messages}

What is the key question?

- Is parental psychological distress during pregnancy associated with lung function and asthma at school age?

What is the bottom line?

- Maternal, but not paternal, psychological distress during pregnancy is associated with an increased risk of asthma and partly lower lung function in children.

Why read on?

- These findings may indicate an intrauterine effect of maternal psychological distress during pregnancy on fetal lung development and respiratory morbidity rather than an effect of unmeasured genetic, social, behavioural or environmental factors.

of the fetal hypothalamic-pituitary-adrenal (HPA) axis, leading to adaptive airway and lung development and asthma. ${ }^{8-10}$ The association of maternal psychological distress during pregnancy with childhood asthma might also be explained by residual confounding factors such as unmeasured genetic, social, behavioural or environmental factors. Since these residual confounding factors are shared by mother and father, while only maternal psychological distress might have intrauterine effects, examining paternal psychological distress during pregnancy in relation to childhood asthma is of importance to account for these residual confounders. ${ }^{311}$ Only a few studies have measured paternal psychological distress, and they found that this is not associated with an increased risk of respiratory symptoms in early childhood, suggesting an intrauterine effect. ${ }^{237}$ To date, the associations of maternal and paternal psychological distress during pregnancy with asthma in later childhood and the association with lung function are not fully clear. $^{12} 13$ We hypothesise that maternal psychological distress during pregnancy, but not paternal psychological distress, is associated with lower lung function and asthma in school-age children, suggesting intrauterine programming for the risk of respiratory disease. 
We therefore examined the association between maternal psychological distress during pregnancy and lung function and asthma in children aged 10 years in a population-based prospective study. We also assessed whether these associations were independent of paternal psychological distress during pregnancy and parental psychological distress after pregnancy.

\section{METHODS}

Design

This study was embedded in the Generation R Study, a population-based prospective cohort study from early fetal life onwards in Rotterdam, the Netherlands. ${ }^{14}$ The study has been approved by the Medical Ethical Committee of the Erasmus MC, University Medical Centre in Rotterdam. Written informed consent was obtained from all parents or legal guardians of the participants. Children from twin pregnancies $(n=185)$, with missing information on maternal psychological distress during pregnancy $(n=1964)$ or missing information on both lung function and asthma $(n=1013)$ were excluded, which left a total of 4231 subjects at age 10 years for the current analyses (online supplementary figure S1).

\section{Maternal and paternal psychological distress}

Maternal and paternal psychological distress was assessed by questionnaires completed in the second trimester of pregnancy and 3 years after pregnancy. Both parents were asked to complete their own questionnaires. At 2 and 6 months after pregnancy, only maternal psychological distress was assessed. We used the Brief Symptom Inventory, a validated 53-item selfreport questionnaire covering a broad spectrum of psychological distress experienced in the last 7 days. ${ }^{15} 16$ A global scale (Global Severity Index (GSI)) and nine symptom scales were defined. ${ }^{17} 18$ The GSI is a measure of the current level or the depth of symptoms and denotes overall psychological distress. Of the symptom scales, we used the depressive and anxiety symptom scale only since, in general, depression and anxiety are considered the most common psychological distress during and after pregnancy. Other subscales encompass less prevalent disorders such as somatisation, obsessive-compulsion, interpersonal sensitivity, hostility, phobia, paranoia and psychoticism. All scales were repeated at 2 months after pregnancy and, at 6 months and 3 years after pregnancy only depressive and anxiety symptom scales were used. All items (53 for the GSI and 6 for both the depressive and anxiety symptom scales) were rated on a 5 -point unidimensional scale ranging from 0 ('not at all') to 4 ('extremely'). Total scores for each scale were calculated by summing the item scores and dividing this by the number of endorsed items. Based on Dutch cut-off values, mothers and fathers were categorised as having clinically relevant psychological distress (no; yes) when having a score of $\geq 0.71$ or $\geq 0.66$ on the global scale, $\geq 0.80$ or $\geq 0.71$ on the depressive symptom scale and $\geq 0.71$ or $\geq 0.65$ on the anxiety symptom scale, respectively. ${ }^{19}$ The Cronbach's alpha, reflecting internal consistency of the different scales, ranged from 0.72 to 0.93 , which is considered acceptable to excellent. Spearman correlations between parental psychological distress during and after pregnancy varied between 0.29 and 0.45 (weak to moderate), and between maternal and paternal psychological distress between 0.12 and 0.23 (very weak to weak).

\section{Lung function and asthma in school-age children}

Lung function was measured by spirometry at the age of 10 years (median 9.7 (5\%-95\% range 9.5-10.3)), as reported earlier. ${ }^{20}$ In short, forced expiratory volume in $1 \mathrm{~s}\left(\mathrm{FEV}_{1}\right)$, forced vital capacity (FVC), $\mathrm{FEV}_{1} / \mathrm{FVC}$ and forced expiratory flow after exhaling $75 \%$ of FVC $\left(\mathrm{FEF}_{75}\right)$ were measured in accordance with the American Thoracic Society (ATS) and European Respiratory Society (ERS) recommendations and converted into sex-, height-, age- and ethnicity-adjusted z-scores according to the Global Lung Initiative reference data. ${ }^{21} 22$ Additionally, we included 281 children whose spirometry did not meet formal reproducibility criteria but who had at least one adequate curve with respect to reach and duration of the plateau according to ATS/ERS criteria. Observed sizes and direction of effect estimates were similar to when these children were excluded. ${ }^{23}$ Current asthma was defined as ever diagnosis of asthma, with either wheezing or any asthma medication use in the past 12 months at the age of 10 years. Information on asthma diagnosis and wheezing was obtained by a parental questionnaire based on the International Study on Asthma and Allergy in Childhood (ISAAC) Questionnaire ("Has your child ever had asthma diagnosed by a doctor?" and "Has your child suffered from attacks of wheezing in the chest in the past 12 months?"). ${ }^{24}$ Information on asthma medication use was obtained during the visit to our research centre.

\section{Covariates}

Information on maternal characteristics included age, parity, ethnicity, educational level, smoking during pregnancy, body mass index at enrolment, history of asthma and atopy and pet keeping, and were obtained from multiple questionnaires during pregnancy. Information on paternal characteristics included age, ethnicity, educational level, smoking before pregnancy, body mass index at enrolment and history of asthma and atopy, and were obtained by a questionnaire during pregnancy. Information on child's sex, gestational age at birth, and birth weight was obtained from midwife and hospital records. Information on child's ethnicity was based on questionnaires during pregnancy, and information on breastfeeding and daycare attendance were obtained by questionnaires in the first year of life. Additional information on confounders can be found in the online supplemental methods.

\section{Statistical analysis}

First, we compared the characteristics of children included in and excluded from our study due to loss to follow-up using Mann-Whitney U tests, t-tests and $\chi^{2}$ tests. Second, we used linear and logistic regression models to study the associations of maternal psychological distress during pregnancy with lung function and current asthma, respectively. Overall psychological distress, depressive and anxiety symptoms were studied separately, and both continuous measures and clinical cut-offs were used. Analyses were adjusted for confounders, which were first selected from the literature and subsequently included in the model if they were associated with maternal psychological distress and lung function or asthma, or if the effect sizes of unadjusted analyses changed $10 \%$ or more after adding a confounder (main model). All confounders selected from the literature met these criteria, and we therefore included all confounders in the model. A directed acyclic graph of the confounders is shown in online supplementary figure S2. Additionally, we added paternal psychological distress during pregnancy to the model to minimise the potential correlated effect of maternal and paternal psychological distress during pregnancy on childhood lung function and asthma, and to assess the individual effect of maternal psychological distress during pregnancy. Next, we added maternal psychological distress 
after pregnancy to the main model as a mediator to disentangle the individual effect of maternal psychological distress during pregnancy on respiratory morbidity of the child. We assessed the change in effect estimates for the associations of maternal psychological distress during pregnancy with lung function and asthma, after additional adjustment for maternal psychological distress after pregnancy, by using the following formulae for percentage change: 100 * Effect estimate $_{\text {full model }}-$ Effect estimate $_{\text {original model }} /\left(\right.$ Effect estimate original model $\left._{\text {e }}\right)$ for continuous lung function measures, and $100 *$ (Effect estimate full model - Effect estimate $\left._{\text {original model }}\right) /\left(\right.$ Effect estimate $\left._{\text {original model }}-1\right)$ for categorical asthma. All other confounders were kept similar to the model with only maternal distress during pregnancy, to ascertain that the change in effect estimate is only due to the additional adjustment for parental psychological distress after pregnancy.

To assess residual confounding effects of unmeasured genetic, social, behavioural or environmental factors, we studied the associations of paternal psychological distress during pregnancy with lung function and asthma. Additionally, we adjusted the associations for paternal psychological distress after pregnancy including change in effect estimates similar to maternal psychological distress. Since parental psychological distress after pregnancy is likely to be correlated to parental distress during pregnancy, adding psychological distress after pregnancy to the model could lead to multicollinearity. Correlations between parental psychological distress during and after pregnancy were, however, below 0.5. Additionally, we assessed the Variance Inflation Factor and tolerance statistics (which are measures for multicollinearity) of the models where parental psychological distress after pregnancy was added to parental psychological distress during pregnancy. Both these measures showed no indication for multicollinearity and inflation of the model. Lastly, we performed a sensitivity analysis focusing on patterns of depressive and anxiety symptoms. In this analysis, depressive and anxiety symptoms during and after pregnancy were combined into the following groups: never, symptoms during pregnancy only, symptoms after pregnancy only, and both symptoms during and after pregnancy. Symptoms after pregnancy reflect psychological distress at either 2,6 or 36 months after pregnancy.

Missing data in covariates (range between 0\% and 33\%), maternal psychological distress after pregnancy and paternal psychological distress during and after pregnancy (range between $26 \%$ and $41 \%$ ) were imputed by multiple imputation using the Markov Chain Monte Carlo method. ${ }^{25}$ We only present the pooled results based on the imputed datasets, since we observed no major differences in the magnitude or direction of the effect when compared with complete case analysis. All measures of association are presented with their 95\% CIs. Statistical analyses were performed using SPSS Version 24.0 for Windows software and $\mathrm{R}$ version 3.4.1.

\section{RESULTS}

\section{Characteristics of subjects}

Parental and child characteristics are shown in tables 1 and 2, respectively. A total of $362(8.6 \%)$ of the participating mothers and $167(3.9 \%)$ of the participating fathers had clinically relevant overall psychological distress. The mean (SD) values for $\mathrm{FEV}_{1}, \mathrm{FVC}, \mathrm{FEV}_{1} / \mathrm{FVC}$ and $\mathrm{FEF}_{75}$ were $2.02(0.30) \mathrm{L}, 2.33(0.36)$ L, $86.72(5.66) \%$ and $1.15(0.35) \mathrm{L} / \mathrm{s}$, respectively. The prevalence of current asthma in children aged 10 years was $5.9 \%$ $(\mathrm{n}=213)$. Most prominently, children lost to follow-up more often had non-European parents and a lower gestational age at birth and birth weight (online supplementary table S1).
Table 1 Characteristics of mothers and fathers

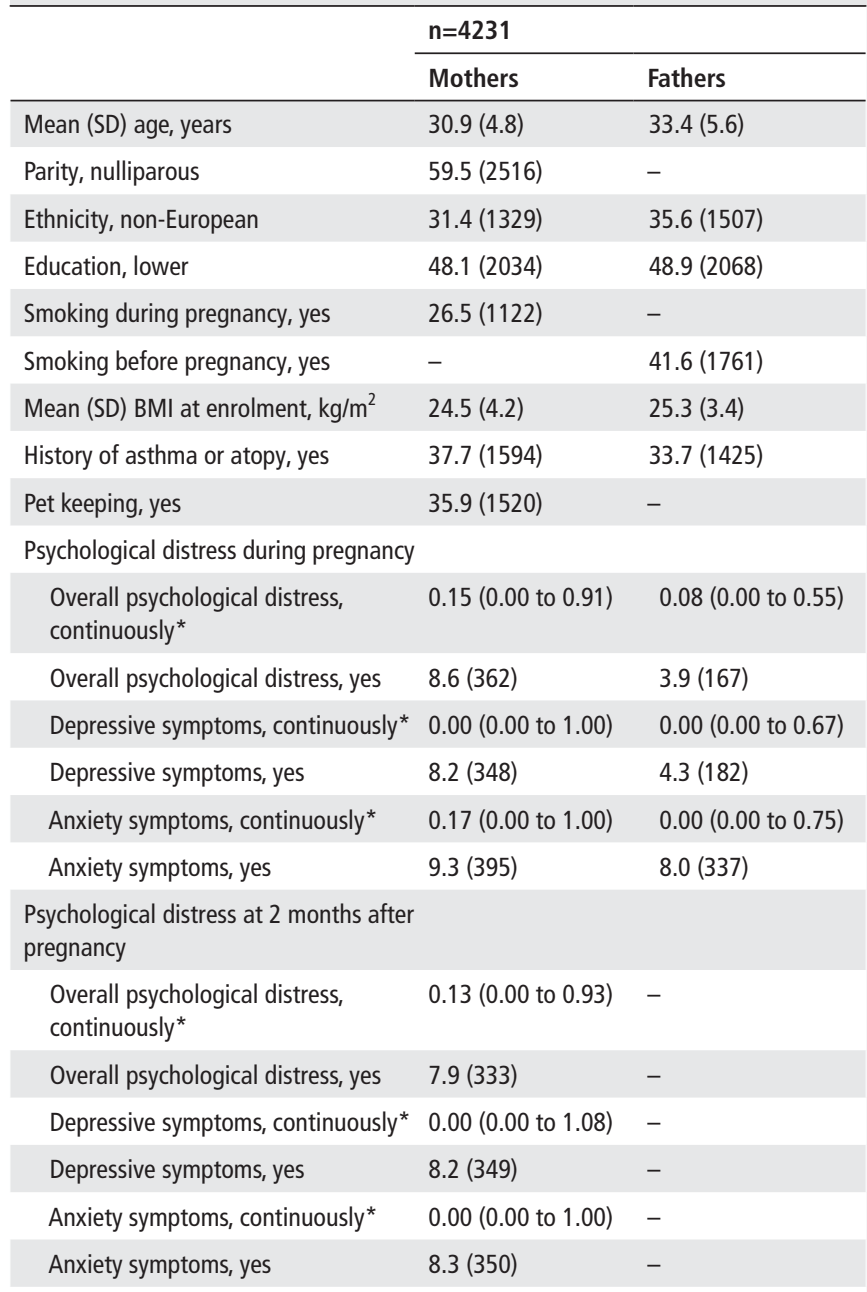

Psychological distress at 6 months after pregnancy

\begin{tabular}{lll}
\hline Depressive symptoms, continuously* & $0.17(0.00$ to 1.17$)$ & - \\
\hline Depressive symptoms, yes & $8.5(358)$ & - \\
\hline Anxiety symptoms, continuously* & $0.00(0.00$ to 1.17$)$ & - \\
\hline Anxiety symptoms, yes & $9.9(417)$
\end{tabular}

Psychological distress at 36 months

after pregnancy

\begin{tabular}{lll} 
Depressive symptoms, continuously* & $0.00(0.00$ to 0.78$)$ & $0.00(0.00$ to 0.68$)$ \\
\hline Depressive symptoms, yes & $5.1(217)$ & $4.4(187)$ \\
\hline Anxiety symptoms, continuously* & $0.00(0.00$ to 0.80$)$ & $0.00(0.00$ to 0.68$)$ \\
\hline Anxiety symptoms, yes & $5.1(214)$ & $7.7(324)$
\end{tabular}

Data on maternal depressive $(n=5)$ and anxiety $(n=6)$ symptoms during pregnancy were missing and not imputed.

Values are means (SD),

*medians (5\%-95\% range), or valid percentages (absolute numbers).

- , not all information was available for both mothers and fathers; BMI, body mass index.

\section{Maternal psychological distress, lung function and asthma}

Unadjusted associations of maternal psychological distress during pregnancy with lung function and asthma in school-age children are shown in online supplementary table S2. After adjustment for confounders and when studied continuously, only maternal overall psychological distress during pregnancy was associated with a lower FVC (z-score difference -0.10 (95\% CI -0.20 to $-0.01)$ ) in their children (table 3). When using clinical cut-offs, 
Table 2 Characteristics of children

\begin{tabular}{|c|c|}
\hline & $1=4231$ \\
\hline Sex, female & $51.2(2166)$ \\
\hline Gestational age at birth, weeks* & $40.1(37.1-42.1)$ \\
\hline Birth weight (g) & $3450(545)$ \\
\hline Ethnicity, non-European & $29.1(1233)$ \\
\hline Ever breastfeeding, yes & $90.9(3847)$ \\
\hline Day care attendance first year, yes & $57.4(2428)$ \\
\hline $\mathrm{FEV}_{1}, \mathrm{~L}$ & $2.02(0.30)$ \\
\hline $\mathrm{FVC}, \mathrm{L}$ & $2.33(0.36)$ \\
\hline $\mathrm{FEV}_{1} / \mathrm{FVC}, \%$ & $86.72(5.66)$ \\
\hline $\mathrm{FEF}_{75}, \mathrm{~L} / \mathrm{s}$ & $1.15(0.35)$ \\
\hline $\mathrm{FEV}_{1}, \mathrm{z}$-score & $0.17(0.97)$ \\
\hline FVC, z-score & $0.21(0.93)$ \\
\hline $\mathrm{FEV}_{1} / \mathrm{FVC}, \mathrm{z}$-score & $-0.10(0.94)$ \\
\hline $\mathrm{FEF}_{75,} z$-score & $0.04(0.91)$ \\
\hline Current asthma, yes & $5.9(213)$ \\
\hline \multicolumn{2}{|c|}{$\begin{array}{l}\text { Lung function measures ( } \mathrm{n}=474) \text { and current asthma }(\mathrm{n}=591) \text { were missing and } \mathrm{n} \\
\text { imputed. } \\
\text { Values are mean (SD), } \\
{ }^{*} \text { medians ( } 5 \%-95 \% \text { range) or valid percentages (absolute numbers). } \\
\mathrm{FEF}_{75^{\prime}} \text { forced expiratory flow after exhaling } 75 \% \text { of } \mathrm{FVC}^{\prime} \mathrm{FEV}_{1} \text {, forced expiratory } \\
\text { volume in } 1 \mathrm{~s} ; \mathrm{FVC} \text {, forced vital capacity. }\end{array}$} \\
\hline
\end{tabular}

only maternal depressive symptoms during pregnancy were associated with a lower $\mathrm{FEV}_{1}$ and FVC $(-0.13$ (95\% CI -0.24 to -0.01$)$ and -0.13 (95\% CI -0.24 to -0.02$)$, respectively). Additional adjustment for paternal psychological distress during pregnancy did not change the direction or magnitude of the effect (table 4). When we additionally adjusted for maternal psychological distress at 2, 6 and 36 months after pregnancy and paternal psychological distress 36 months after pregnancy, the size and direction of the associations of depressive symptoms with a lower $\mathrm{FEV}_{1}$ and FVC remained unchanged, except when adjusting for maternal psychological distress at 2 months (table 5). The percentage changes in effect estimates were, however, non-significant (data not shown).

Maternal overall psychological distress and depressive and anxiety symptoms during pregnancy were all associated with an increased risk of current asthma in their children, both continuously and when using clinical cut-offs (range OR 1.46 (95\% CI 1.12 to 1.90 ) to 1.91 (95\% CI 1.26 to 2.91$)$ ) (table 3 ). When we additionally adjusted for paternal psychological distress during pregnancy, the associations of maternal psychological distress with childhood asthma remained essentially unchanged (table 4). Additional adjustment for maternal psychological distress at 2, 6 and 36 months after pregnancy did not change the direction or magnitude of the effect estimates for the association with asthma, including a non-significant percentage change in effect estimates (table 5). Analysis of patterns of psychological distress showed that mostly depressive or anxiety symptoms both during and after pregnancy are associated with an increased risk of asthma in children (online supplementary table S3). Separating the confounders into three different groups, including lifestyle and health-related factors, socioeconomic factors and birth and early childhood factors, showed no differences in size or direction of effect estimates of the associations of maternal psychological distress with asthma in their children (online supplementary table S4). However, lifestyle and health-related factors seemed to account for attenuation of the effect of maternal psychological distress with $\mathrm{FEF}_{75}$ only.

\section{Paternal psychological distress, lung function and asthma}

Paternal psychological distress during pregnancy was not associated with lung function or asthma in their children (table 6). The associations of paternal psychological distress during pregnancy with lung function and asthma remained essentially unchanged after additional adjustment for paternal psychological distress at 36 months after pregnancy (online supplementary table S5). Only the associations of clinical cut-offs of anxiety with FEV and FVC became significant, as opposed to before adjustment for paternal psychological distress after birth $(-0.15(95 \% \mathrm{CI}$ -0.29 to -0.00$)$ and $-0.14(95 \%$ CI -0.29 to -0.00$)$, respectively). However, the percentage changes for these and other

Table 3 Associations of maternal psychological distress during pregnancy with lung function and asthma in children at age 10 years

\begin{tabular}{|c|c|c|c|c|c|c|}
\hline & $\mathrm{N}$ & $\begin{array}{l}\text { FEV }_{1} \\
\text { Z-score }(95 \% \mathrm{Cl}) \\
\mathrm{n}=3757\end{array}$ & $\begin{array}{l}\text { FVC } \\
\text { Z-score }(95 \% \mathrm{Cl}) \\
\mathrm{n}=3757\end{array}$ & $\begin{array}{l}\mathrm{FEV}_{1} / \mathrm{FVC} \\
\mathrm{Z} \text {-score }(95 \% \mathrm{Cl}) \\
\mathrm{n}=3757\end{array}$ & $\begin{array}{l}\mathrm{FEF}_{75} \\
\mathrm{Z} \text {-score }(95 \% \mathrm{Cl}) \\
\mathrm{n}=3757\end{array}$ & $\begin{array}{l}\text { Current asthma } \\
\text { OR }(95 \% \mathrm{Cl}) \\
\mathrm{n}=3640\end{array}$ \\
\hline \multicolumn{7}{|l|}{ Maternal psychological distress } \\
\hline Overall psychological distress & 4231 & & & & & \\
\hline Per 1-unit increase & & $-0.07(-0.17$ to 0.03$)$ & $-0.10(-0.20 \text { to }-0.01)^{*}$ & $0.04(-0.06$ to 0.13$)$ & $0.01(-0.08$ to 0.10$)$ & $1.83(1.30 \text { to } 2.59)^{* *}$ \\
\hline Clinical cut-off & & $-0.02(-0.14$ to 0.10$)$ & $-0.07(-0.14$ to 0.01$)$ & $0.03(-0.05$ to 0.10$)$ & $0.02(-0.05$ to 0.09$)$ & 1.91 (1.26 to 2.91$)^{* *}$ \\
\hline Depressive symptoms & 4225 & & & & & \\
\hline Per 1-unit increase & & $-0.05(-0.12$ to 0.03$)$ & $-0.07(-0.14$ to 0.01$)$ & $0.03(-0.04$ to 0.11$)$ & $0.02(-0.05$ to 0.09$)$ & $1.46(1.12 \text { to } 1.90)^{* *}$ \\
\hline Clinical cut-off & & $-0.13(-0.24 \text { to }-0.01)^{*}$ & $-0.13(-0.24 \text { to }-0.02)^{*}$ & $-0.01(-0.12$ to 0.11$)$ & $-0.03(-0.14$ to 0.08$)$ & $1.84(1.21 \text { to } 2.80)^{* *}$ \\
\hline Anxiety symptoms & 4226 & & & & & \\
\hline Per 1-unit increase & & $-0.01(-0.09$ to 0.07$)$ & $-0.03(-0.10$ to 0.05$)$ & $0.01(-0.07$ to 0.09$)$ & $-0.00(-0.07$ to 0.07$)$ & 1.55 (1.19 to 2.02$)^{* *}$ \\
\hline Clinical cut-off & & $-0.01(-0.12$ to 0.10$)$ & $0.01(-0.09$ to 0.11$)$ & $-0.05(-0.16$ to 0.06$)$ & $-0.05(-0.15$ to 0.05$)$ & 1.64 (1.09 to 2.47$)^{*}$ \\
\hline
\end{tabular}

Values are z-scores or odds ratios (OR) with $95 \%$ Cls from linear or logistic regression models, respectively.

Maternal psychological distress is treated as a continuous variable (per 1-unit increase) or a dichotomous variable based on clinical cut-offs (no; yes, where 'no' was the reference category).

The main models were adjusted for maternal age, parity, education level, smoking during pregnancy, body mass index at enrolment, history of asthma or atopy and pet keeping, and child's sex, gestational age at birth, birth weight, ethnicity, breastfeeding and daycare attendance.

${ }^{*} \mathrm{P}<0.05,{ }^{* *} \mathrm{P}<0.01$.

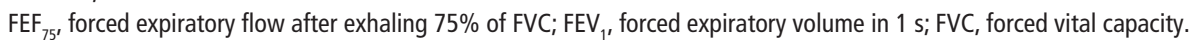




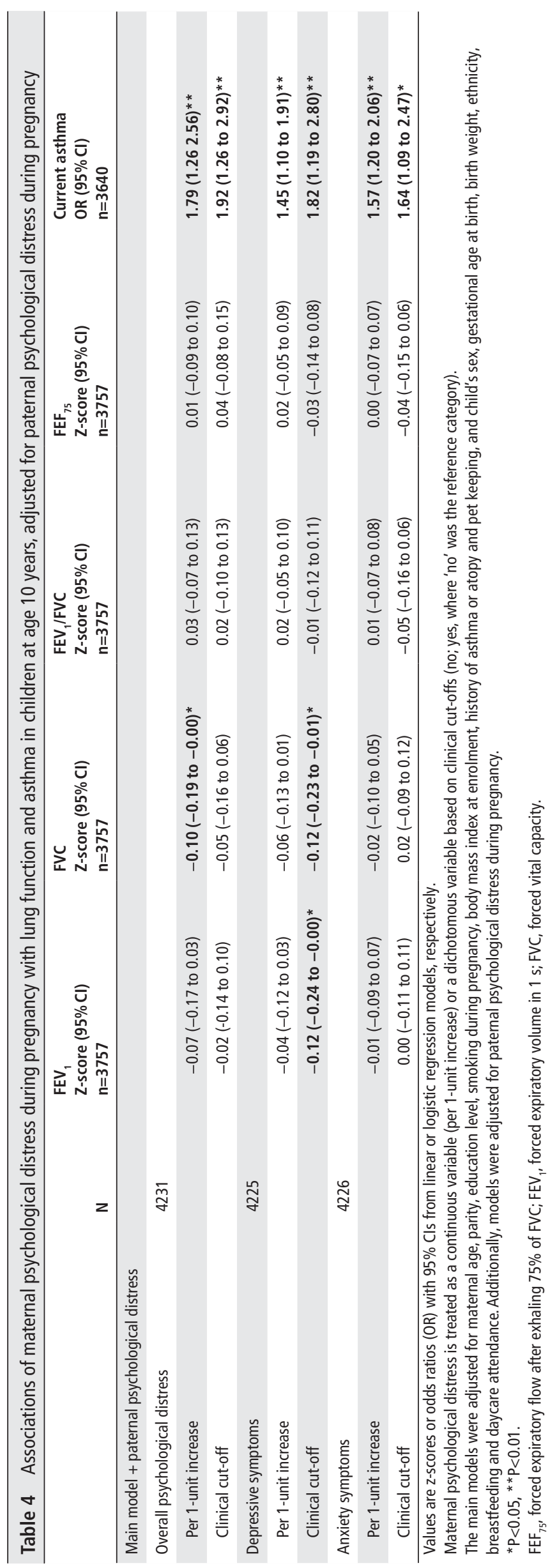

associations, when adding paternal psychological distress after pregnancy to the model, were non-significant (online supplementary table S6).

\section{DISCUSSION}

The results of this population-based prospective cohort study showed that children of mothers with overall psychological distress and depressive or anxiety symptoms during pregnancy had an increased risk of asthma at the age of 10 years. Children of mothers with overall psychological distress when measured continuously had a lower FVC, and children of mothers with depressive symptoms when using clinical cut-offs had a lower $\mathrm{FEV}_{1}$ and FVC. These findings were mostly independent of paternal psychological distress during pregnancy, and maternal and paternal psychological distress after pregnancy. The strongest effects were found for mothers who experienced psychological distress both during and after pregnancy. Although the effects of distress during pregnancy only were not significant, this is most likely due to the power of the study, given that the effects estimates are in the same range as for the main analysis. Paternal psychological distress during pregnancy was not associated with an increased risk of lower lung function or asthma. Our results may indicate an intrauterine effect of maternal psychological distress during pregnancy on fetal lung development and respiratory morbidity rather than an effect of unmeasured genetic, social, behavioural or environmental factors.

\section{Comparison with previous studies}

Studies examining the association of maternal psychological distress with childhood lung function are scarce. One study found that high prenatal but also postnatal maternal psychological distress, measured as negative life events, was associated with a lower $\mathrm{FEV}_{1}$, FVC and mid-expiratory flow $\left(\mathrm{FEF}_{25-75}\right)$ but not $\mathrm{FEV}_{1} / \mathrm{FVC}$ at age 7 years. ${ }^{26}$ This is in line with our study, where we found that the association of maternal psychological distress with a lower $\mathrm{FEV}_{1}$ and FVC remains present in later childhood. These significant findings, compared with the more robust findings for the asthma outcome, are few and effect sizes are small from a clinical perspective but of importance from an aetiological perspective. Studies examining the association of maternal psychological stress during pregnancy with wheezing and asthma in children have been summarised in two recent meta-analyses. ${ }^{56}$ Both meta-analyses focused mostly on wheezing and asthma until preschool age, with only a few studies that measured respiratory outcomes after the age of 6 years. One meta-analysis including 10 studies found that maternal psychological stress during pregnancy was associated with a 1.56 -fold increased risk of any respiratory morbidity in the children. ${ }^{5}$ When the associations with asthma and wheezing were studied separately, the effect sizes were approximately in the same range, although the latter showed high heterogeneity. The other more recent meta-analysis including 24 studies demonstrated that any maternal psychological stress during pregnancy was associated with a 1.13 -fold increased risk of asthma in the children. ${ }^{6}$ This was most likely driven by anxiety during pregnancy, since only this association was significant when different types of psychological distress were studied separately. Anxiety, depression and negative life events during pregnancy were all associated with an increased risk of wheezing (OR or RR 1.19, 1.74 and 1.23, respectively). Our study shows that maternal stress during pregnancy is associated with an increased risk of asthma even at a later age, with effect sizes within the same range. Additionally, we demonstrated that these results are independent of paternal 
Table 5 Associations of maternal psychological distress with lung function and asthma in children at age 10 years, adjusted for maternal psychological distress at 2,6 and 36 months after pregnancy and paternal psychological distress at 36 months after pregnancy

\begin{tabular}{|c|c|c|c|c|c|c|}
\hline & $\mathrm{N}$ & $\begin{array}{l}\mathrm{FEV}_{1} \\
\mathrm{Z} \text {-score }(95 \% \mathrm{Cl}) \\
\mathrm{n}=3757\end{array}$ & $\begin{array}{l}\text { FVC } \\
Z \text {-score }(95 \% \mathrm{Cl}) \\
\mathrm{n}=3757\end{array}$ & $\begin{array}{l}\mathrm{FEV}_{1} / \mathrm{FVC} \\
\mathrm{Z}_{\mathrm{score}}(95 \% \mathrm{Cl}) \\
\mathrm{n}=3757\end{array}$ & $\begin{array}{l}\mathrm{FEF}_{75} \\
\mathrm{Z} \text {-score }(95 \% \mathrm{Cl}) \\
\mathrm{n}=3757\end{array}$ & $\begin{array}{l}\text { Current asthma } \\
\text { OR }(95 \% \mathrm{Cl}) \\
\mathrm{n}=3640\end{array}$ \\
\hline \multicolumn{7}{|l|}{$\begin{array}{l}\text { Main model + maternal } \\
\text { psychological distress at } 2 \\
\text { months }\end{array}$} \\
\hline $\begin{array}{l}\text { Overall psychological } \\
\text { distress }\end{array}$ & 4231 & & & & & \\
\hline Per 1-unit increase & & $-0.03(-0.15$ to 0.09$)$ & $-0.06(-0.18$ to 0.05$)$ & 0.05 (-0.08 to 0.17$)$ & 0.05 (-0.07 to 0.18$)$ & 2.01 (1.26 to 3.22$)^{* *}$ \\
\hline Clinical cut-off & & $0.01(-0.12$ to 0.14$)$ & $-0.02(-0.15$ to 0.10$)$ & $0.02(-0.11$ to 0.14$)$ & 0.06 (-0.06 to 0.18$)$ & $2.11(1.30 \text { to } 3.43)^{* *}$ \\
\hline Depressive symptoms & 4226 & & & & & \\
\hline Per 1-unit increase & & $-0.02(-0.11$ to 0.06$)$ & $-0.05(-0.13$ to 0.03$)$ & $0.04(-0.05$ to 0.12$)$ & $0.04(-0.04$ to 0.13$)$ & 1.37 (1.00 to 1.88$)$ \\
\hline Clinical cut-off & & $-0.12(-0.23$ to 0.01$)$ & $-0.10(-0.21$ to 0.02$)$ & $-0.04(-0.16$ to 0.09$)$ & $-0.04(-0.16$ to 0.08$)$ & $1.87(1.18 \text { to } 2.97)^{* *}$ \\
\hline Anxiety symptoms & 4225 & & & & & \\
\hline Per 1-unit increase & & 0.00 (-0.09 to 0.09$)$ & $-0.01(-0.10$ to 0.08$)$ & 0.00 (-0.09 to 0.10$)$ & 0.00 (-0.09 to 0.09 ) & $1.70(1.20 \text { to } 2.41)^{* *}$ \\
\hline Clinical cut-off & & $-0.01(-0.12$ to 0.11$)$ & 0.02 (-0.09 to 0.13$)$ & $-0.06(-0.18$ to 0.05$)$ & $-0.05(-0.16$ to 0.06$)$ & $1.72(1.09 \text { to } 2.72)^{*}$ \\
\hline
\end{tabular}

Main model + maternal

psychological distress at 6

months

\begin{tabular}{|c|c|c|c|c|c|c|}
\hline Depressive symptoms & 4226 & & & & & \\
\hline Per 1-unit increase & & $-0.05(-0.14$ to 0.04$)$ & $-0.06(-0.14$ to 0.03$)$ & $0.00(0.08$ to 0.09$)$ & $0.01(-0.08$ to 0.09$)$ & 1.29 (0.93 to 1.78$)$ \\
\hline Clinical cut-off & & $-0.13(-0.26 \text { to }-0.01)^{*}$ & $-0.13(-0.24 \text { to }-0.01)^{*}$ & $-0.02(-0.15$ to 0.10$)$ & $-0.04(-0.16$ to 0.08$)$ & 1.58 (0.99 to 2.54$)$ \\
\hline Anxiety symptoms & 4225 & & & & & \\
\hline Per 1-unit increase & & $-0.04(-0.13$ to 0.04$)$ & $-0.04(-0.13$ to 0.04$)$ & $-0.02(-0.11$ to 0.07$)$ & -0.02 ( -0.11 to 0.06$)$ & $1.61(1.16 \text { to } 2.22)^{* *}$ \\
\hline Clinical cut-off & & $-0.02(-0.13$ to 0.09$)$ & $-0.01(-0.11$ to 0.12$)$ & $-0.07(-0.18$ to 0.05$)$ & $-0.06(-0.17$ to 0.05$)$ & $1.59(1.02 \text { to } 2.48)^{*}$ \\
\hline
\end{tabular}

Main model + maternal

psychological distress at 36

months

\begin{tabular}{|c|c|c|c|c|c|c|}
\hline Depressive symptoms & 4226 & & & & & \\
\hline Per 1-unit increase & & $-0.05(-0.13$ to 0.04$)$ & $-0.07(-0.15$ to 0.01$)$ & $0.03(-0.05$ to 0.11$)$ & $0.03(-0.06$ to 0.11$)$ & $1.38(1.02 \text { to } 1.86)^{*}$ \\
\hline Clinical cut-off & & $-0.12(-0.24 \text { to }-0.00)^{*}$ & $-0.12(-0.24 \text { to }-0.01)^{*}$ & $-0.01(-0.13$ to 0.11$)$ & $-0.02(-0.14$ to 0.09$)$ & $1.78(1.14 \text { to } 2.78)^{*}$ \\
\hline Anxiety symptoms & 4225 & & & & & \\
\hline Per 1-unit increase & & $-0.03(-0.11$ to 0.06$)$ & $-0.03(-0.12$ to 0.05$)$ & $-0.00(-0.09$ to 0.08$)$ & $-0.01(-0.09$ to 0.07$)$ & $1.43(1.05 \text { to } 1.95)^{*}$ \\
\hline Clinical cut-off & & $-0.01(-0.12$ to 0.10$)$ & $0.01(-0.10$ to 0.12$)$ & $-0.06(-0.17$ to 0.06$)$ & $-0.05(-0.15$ to 0.06$)$ & 1.52 (0.99 to 2.34$)$ \\
\hline \multicolumn{7}{|l|}{$\begin{array}{l}\text { Main model + paternal } \\
\text { psychological distress at } 36 \\
\text { months }\end{array}$} \\
\hline Depressive symptoms & 4226 & & & & & \\
\hline Per 1-unit increase & & $-0.05(-0.12$ to 0.03$)$ & $-0.07(-0.14$ to 0.00$)$ & $0.03(-0.05$ to 0.11$)$ & $0.02(-0.05$ to 0.10$)$ & $1.41(1.07 \text { to } 1.86)^{*}$ \\
\hline Clinical cut-off & & $-0.13(-0.24 \text { to }-0.01)^{*}$ & $-0.13(-0.24 \text { to }-0.02)^{*}$ & $-0.00(-0.12$ to 0.11$)$ & $-0.03(-0.14$ to 0.08$)$ & $1.78(1.16 \text { to } 2.74)^{*}$ \\
\hline Anxiety symptoms & 4225 & & & & & \\
\hline Per 1-unit increase & & $-0.02(-0.09$ to 0.06$)$ & $-0.03(-0.11$ to 0.04$)$ & $0.01(-0.07$ to 0.09$)$ & $-0.00(-0.08$ to 0.07$)$ & $1.51(1.15 \text { to } 1.99)^{* *}$ \\
\hline Clinical cut-off & & $-0.01(-0.12$ to 0.10$)$ & $0.01(-0.10$ to 0.11$)$ & $-0.05(-0.16$ to 0.06$)$ & $-0.05(-0.15$ to 0.05$)$ & $1.60(1.06 \text { to } 2.41)^{*}$ \\
\hline
\end{tabular}

Values are z-scores or odds ratios (OR) with $95 \%$ Cls from linear or logistic regression models, respectively.

Maternal psychological distress is treated as a continuous variable (per 1-unit increase) or a dichotomous variable based on clinical cut-offs (no; yes, where 'no' was the reference category).

The main models were adjusted for maternal age, parity, education level, smoking during pregnancy, body mass index at enrolment, history of asthma or atopy and pet keeping, and child's sex, gestational age at birth, birth weight, ethnicity, breastfeeding and daycare attendance. Additionally, models were adjusted for maternal psychological distress at 2,6 or 36 months after pregnancy or for paternal psychological distress at 36 months after pregnancy. At 6 and 36 months after pregnancy, not all subscales were measured and therefore overall psychological distress could not be included at these time points.

${ }^{*} \mathrm{P}<0.05,{ }^{* *} \mathrm{P}<0.01$.

$\mathrm{FEF}_{75^{\prime}}$, forced expiratory flow after exhaling $75 \%$ of $\mathrm{FVC}_{;} \mathrm{FEV}_{1^{\prime}}$, forced expiratory volume in $1 \mathrm{~s} ; \mathrm{FVC}$, forced vital capacity.

psychological distress during pregnancy and parental psychological distress after pregnancy, which was not studied in these meta-analyses.

\section{Possible mechanisms}

Our results suggest that intrauterine mechanisms may underlie 


\begin{tabular}{|c|c|c|c|c|c|c|}
\hline & $\mathrm{N}$ & $\begin{array}{l}\mathrm{FEV}_{1} \\
\mathrm{Z} \text {-score }(95 \% \mathrm{Cl}) \\
\mathrm{n}=3757\end{array}$ & $\begin{array}{l}\text { FVC } \\
Z \text { Z-score }(95 \% \text { Cl) } \\
n=3757\end{array}$ & $\begin{array}{l}\mathrm{FEV}_{1} / \mathrm{FVC} \\
\mathrm{Z} \text {-score }(95 \% \mathrm{Cl}) \\
\mathrm{n}=3757\end{array}$ & $\begin{array}{l}\text { FEF }_{75} \\
\text { Z-score }(95 \% \mathrm{Cl}) \\
\mathrm{n}=3757\end{array}$ & $\begin{array}{l}\text { Current asthma } \\
\text { OR }(95 \% \mathrm{Cl}) \\
\mathrm{n}=3640\end{array}$ \\
\hline \multicolumn{7}{|l|}{ Paternal psychological distress } \\
\hline Overall psychological distress & 4231 & & & & & \\
\hline Per 1-unit increase & & $0.00(-0.18$ to 0.18$)$ & $-0.05(-0.23$ to 0.13$)$ & $0.07(-0.08$ to 0.22$)$ & $0.03(-0.13$ to 0.19$)$ & 1.13 (0.56 to 2.26$)$ \\
\hline Depressive symptoms & 4231 & & & & & \\
\hline Per 1-unit increase & & $-0.02(-0.18$ to 0.14$)$ & $-0.04(-0.19$ to 0.11$)$ & $0.02(-0.10$ to 0.14$)$ & $-0.01(-0.16$ to 0.13$)$ & 1.04 (0.59 to 1.85$)$ \\
\hline Clinical cut-off & & $-0.07(-0.30$ to 0.16$)$ & $-0.07(-0.30$ to 0.16$)$ & $-0.02(-0.20$ to 0.17$)$ & $-0.06(-0.25$ to 0.13$)$ & 1.04 (0.43 to 2.53$)$ \\
\hline Anxiety symptoms & 4231 & & & & & \\
\hline Per 1-unit increase & & $-0.01(-0.17$ to 0.15$)$ & $-0.03(-0.19$ to 0.14$)$ & $0.02(-0.10$ to 0.14$)$ & $-0.01(-0.14$ to 0.13$)$ & 0.85 (0.46 to 1.58$)$ \\
\hline
\end{tabular}

Values are z-scores or odds ratios (OR) with $95 \% \mathrm{Cls}$ from linear or logistic regression models, respectively.

Paternal psychological distress is treated as a continuous variable (per 1-unit increase) or a dichotomous variable based on clinical cut-offs (no; yes, where 'no' was the reference category).

The models were adjusted for maternal age, parity, education level, smoking during pregnancy, body mass index at enrolment, history of asthma or atopy and pet keeping, and child's sex, gestational age at birth, birth weight, ethnicity, breastfeeding and daycare attendance and maternal psychological distress during pregnancy. $\mathrm{FEF}_{75}$, forced expiratory flow after exhaling $75 \%$ of FVC; $\mathrm{FEV}_{1}$, forced expiratory volume in $1 \mathrm{~s}$; FVC, forced vital capacity.

the associations of maternal psychological distress with childhood lung function and asthma in their children, rather than unmeasured genetic, social, behavioural or environmental factors. One potential intrauterine mechanism is excess glucocorticoid production due to maternal psychological distress, which could lead to impaired development of the fetal HPA axis. $^{8}$ Additionally, maternal psychological distress could influence the stimulation of corticotrophin releasing hormone $(\mathrm{CRH})$ secretion, which results in increased $\mathrm{CRH}$ levels in the fetal circulation and could overstimulate the fetal HPA axis. Moreover, glucocorticoid-regulated genes are key to fetal lung development, especially during the first and second trimesters of pregnancy. ${ }^{9}$ Any disruption in this process could lead to developmental adaptations of the lungs and, hence, altered lung function.

Epigenetics has also been proposed as a possible mechanism. It has been shown that maternal depression and anxiety are associated with methylation of the glucocorticoid receptor gene NR3C1 in cord blood. ${ }^{27}$ Additionally, one study demonstrated an association of prenatal maternal stress with differently methylated regions related to the HPA axis, immune responses and lung organogenesis. ${ }^{28}$

Finally, oxidative stress and the microbiome have been speculated as possible underlying mechanisms for the association of maternal psychological distress during pregnancy with lung function and asthma in their offspring. ${ }^{29-31}$ However, these mechanisms should be studied in more detail.

\section{Strengths and limitations of the study}

The major strength of this study is the use of a large populationbased prospective cohort with follow-up until school age. In our study, we used a well-known measure of psychological distress frequently used in epidemiological studies to assess psychological distress at multiple time points, both continuously and with clinical cut-offs. Additionally, we adjusted for multiple possible confounding factors and considered paternal psychological distress to assess unmeasured confounding factors. Some methodological limitations should be discussed. As in any prospective cohort study, our population was subject to loss to follow-up. Of those with singleton liveborn children with consent at age 10 years, $73 \%$ responded to the questionnaire measuring psychological distress. This loss to follow-up could lead to bias if the associations of maternal psychological distress during pregnancy with lung function and asthma were different between those included and those not included in the study. This could lead to both an underestimation or an overestimation of the association and, although this is unlikely, it cannot be excluded. ${ }^{32}$ Additionally, self-reporting of exposure and outcomes could potentially lead to information bias which could result in misclassification. The use of validated questionnaires could have minimised the information bias. The prevalence of asthma seems relatively low, but lies within national and worldwide prevalence rates. ${ }^{33} 34$ We consider self-reported asthma as truly asthma because questions were based on the validated ISAAC questionnaire, and wheezing or asthma medication use in the past 12 months was used to better define current asthma. Also, the results were similar when we used persistent asthma as an outcome (data not shown). Moreover, psychological distress was measured only once during pregnancy. Hence, we do not know whether maternal psychological distress varied in intensity or persistence throughout pregnancy. However, one study that measured maternal anxiety at two time points during pregnancy demonstrated associations with asthma that were comparable for both time points, suggesting that timing in pregnancy seems to have a less prominent role in prenatal programming. ${ }^{2}$ Further studies are needed to confirm these results. Lastly, although we adjusted for numerous confounders, we might not have had information on all possible confounding factors such as maternal or child genetic predisposition for psychological stress disorders or childhood exposures such as air pollution. Although some of their effects might be minimal and are highly correlated with presently used confounders, they could potentially have a substantial effect given the relatively small prevalence of asthma.

\section{CONCLUSION}

Our results suggest a possible intrauterine effect of maternal psychological distress during pregnancy on the risk of asthma and partly lower lung function in children at the age of 10 years. The results were independent of maternal psychological distress after pregnancy and paternal psychological distress during 
pregnancy and after pregnancy. Further studies are needed to explore underlying mechanisms.

\section{Author affiliations}

'The Generation R Study Group, Erasmus Medical Center, Rotterdam, The Netherlands

${ }^{2}$ Department of Pediatrics, Division of Respiratory Medicine and Allergology, Erasmus Medical Center, Rotterdam, The Netherlands

${ }^{3}$ Department of Pediatrics, Erasmus Medical Center, Rotterdam, The Netherlands ${ }^{4}$ Department of Pediatrics, Division of Neonatology, Erasmus Medical Center, Rotterdam, Zuid-Holland, The Netherlands

${ }^{5}$ Department of Child and Adolescent Psychiatry, Erasmus Medical Center, Rotterdam, The Netherlands

${ }^{6}$ Department of Epidemiology, Erasmus Medical Center, Rotterdam, The Netherlands 7 Department of Social and Behavioural Science, Harvard University T H Chan School of Public Health, Boston, Massachusetts, USA

${ }^{8}$ Department of Psychology, Education and Child Studies, Erasmus Universiteit Rotterdam, Rotterdam, The Netherlands

Acknowledgements The Generation R Study is conducted by the Erasmus Medical Center in close collaboration with the School of Law and the Faculty of Social Sciences at the Erasmus University, Rotterdam, the Municipal Health Service, Rotterdam area and the Stichting Trombosedienst and Artsenlaboratorium Rijnmond (Star-MDC), Rotterdam. We gratefully acknowledge the contribution of children and their parents, general practitioners, hospitals, midwives and pharmacies in Rotterdam.

Contributors ERvM, GS and LD contributed to the conception and design, acquisition of data, analyses and interpretation of the data, drafted the article, revised it critically for important intellectual content and gave final approval of the version to be published. VJ, JCdJ, IR, HT and HEM contributed to the conception and design, acquisition of data, revised the drafted manuscript critically for important intellectual content and gave final approval of the version to be published.

Funding The Generation R Study is made possible by financial support from the Erasmus Medical Center, Rotterdam, the Erasmus University Rotterdam and the Netherlands Organization for Health Research and Development. LD received funding from the co-funded programme ERA-Net on Biomarkers for Nutrition and Health (ERA HDHL) (ALPHABET project, Horizon 2020 (grant agreement no 696295; 2017), ZonMW, The Netherlands (no 529051014; 2017)). HT was supported by a grant from the Netherlands Organization for Scientific Research (NWO 016. $\mathrm{VICI}$ 170.200). The project received funding from the European Union's Horizon 2020 research and innovation programme (LifeCycle project, grant agreement no 733209; 2016). The researchers are independent from the funders. The study sponsors had no role in the study design, data analysis, interpretation of data or writing of this report.

Competing interests None declared.

Patient consent for publication Not required.

Provenance and peer review Not commissioned; externally peer reviewed.

Data availability statement No data are available.

Open access This is an open access article distributed in accordance with the Creative Commons Attribution 4.0 Unported (CC BY 4.0) license, which permits others to copy, redistribute, remix, transform and build upon this work for any purpose, provided the original work is properly cited, a link to the licence is given, and indication of whether changes were made. See: https://creativecommons.org/ licenses/by/4.0/.

ORCID iDs

Evelien R van Meel http://orcid.org/0000-0002-0826-9931

Liesbeth Duijts http://orcid.org/0000-0001-6731-9452

\section{REFERENCES}

1 Duijts L. Fetal and infant origins of asthma. Eur J Epidemiol 2012;27:5-14.

2 Cookson H, Granell R, Joinson C, et al. Mothers' anxiety during pregnancy is associated with asthma in their children. J Allergy Clin Immunol 2009;123:847-53.

3 Guxens M, Sonnenschein-van der Voort AMM, Tiemeier H, et al. Parental psychological distress during pregnancy and wheezing in preschool children: the Generation $\mathrm{R}$ Study. J Allergy Clin Immunol 2014;133:59-67.

4 Wright RJ. Perinatal stress and early life programming of lung structure and function. Biol Psychol 2010;84:46-56.

5 van de Loo KFE, van Gelder MMHJ, Roukema J, et al. Prenatal maternal psychological stress and childhood asthma and wheezing: a meta-analysis. Eur Respir J 2016;47:133-46.
6 Flanigan C, Sheikh A, DunnGalvin A, et al. Prenatal maternal psychosocial stress and offspring's asthma and allergic disease: a systematic review and meta-analysis. Clin Exp Allergy 2018;48:403-14.

7 Brew BK, Gong T, Williams DM, et al. Using fathers as a negative control exposure to test the developmental origins of health and disease hypothesis: a case study on maternal distress and offspring asthma using Swedish register data. Scand I Public Health 2017:45:36-40.

8 Reynolds RM. Glucocorticoid excess and the developmental origins of disease: two decades of testing the hypothesis--2012 Curt Richter Award Winner. Psychoneuroendocrinology 2013;38:1-11.

9 Sharma S, Kho AT, Chhabra D, et al. Glucocorticoid genes and the developmental origins of asthma susceptibility and treatment response. Am J Respir Cell Mol Biol 2015:52:543-53.

10 Glover V. Prenatal stress and its effects on the fetus and the child: possible underlying biological mechanisms. Adv Neurobiol 2015;10:269-83.

11 Smith GD. Assessing intrauterine influences on offspring health outcomes: can epidemiological studies yield robust findings? Basic Clin Pharmacol Toxicol 2008; 102:245-56

12 de Marco R, Pesce G, Girardi P, et al. Foetal exposure to maternal stressful events increases the risk of having asthma and atopic diseases in childhood. Pediatr Allergy Immunol 2012;23:724-9.

13 Hartwig IRV, Sly PD, Schmidt LA, et al. Prenatal adverse life events increase the risk for atopic diseases in children, which is enhanced in the absence of a maternal atopic predisposition. J Allergy Clin Immunol 2014;134:160-9.

14 Kooijman MN, Kruithof CJ, van Duijn CM, et al. The Generation R Study: design and cohort update 2017. Eur J Epidemiol 2016:31:1243-64.

15 Derogatis LR. BSI brief symptom inventory, administration, scoring, and procedures manual. 4th edn. Minneapolis, MN: National Computer Systems, 1993.

16 de Beurs E. Handleiding bij de brief symptom inventory (BSI). Leiden: PITS B.V, 2004

17 von Hertzen LC. Maternal stress and T-cell differentiation of the developing immune system: possible implications for the development of asthma and atopy. J Allergy Clin Immunol 2002;109:923-8.

18 den Hollander-Giijsman ME, de Beurs E, van der Wee NJA, et al. Distinguishing between depression and anxiety: a proposal for an extension of the tripartite model. Eur Psychiatry 2010;25:197-205.

19 Wardenaar KJ, van Veen T, Giltay EJ, et al. Development and validation of a 30-item short adaptation of the Mood and Anxiety Symptoms Questionnaire (MASQ). Psychiatry Res 2010;179:101-6.

20 van Meel ER, de Jong M, Elbert NJ, et al. Duration and exclusiveness of breastfeeding and school-age lung function and asthma. Ann Allergy Asthma Immunol 2017:119:21-6.

21 Quanjer PH, Stanojevic S, Cole TJ, et al. Multi-ethnic reference values for spirometry for the 3-95-yr age range: the global lung function 2012 equations. Eur Respir J 2012:40:1324-43.

22 Miller MR, Hankinson J, Brusasco V, et al. Standardisation of spirometry. Eur Respir J 2005;26:319-38

23 den Dekker HT, Jaddoe VWV, Reiss IK, et al. Fetal and infant growth patterns and risk of lower lung function and asthma. The Generation R Study. Am J Respir Crit Care Med 2018;197:183-92.

24 Asher MI, Keil U, Anderson HR, et al. International Study of Asthma and Allergies in Childhood (ISAAC): rationale and methods. Eur Respir J 1995;8:483-91.

25 Spratt M, Carpenter J, Sterne JAC, et al. Strategies for multiple imputation in longitudinal studies. Am J Epidemiol 2010;172:478-87.

26 Lee AG, Chiu Y-HM, Rosa MJ, et al. Association of prenatal and early childhood stress with reduced lung function in 7-year-olds. Ann Allergy Asthma Immunol 2017;119:153-9

27 Oberlander TF, Weinberg J, Papsdorf $\mathrm{M}$, et al. Prenatal exposure to maternal depression, neonatal methylation of human glucocorticoid receptor gene (NR3C1) and infant cortisol stress responses. Epigenetics 2008:3:97-106.

28 Trump S, Bieg M, Gu Z, et al. Prenatal maternal stress and wheeze in children: nove insights into epigenetic regulation. Sci Rep 2016;6:28616.

29 Manti S, Marseglia L, D'Angelo G, et al. "Cumulative stress": the effects of maternal and neonatal oxidative stress and oxidative stress-inducible genes on programming of atopy. Oxid Med Cell Longev 2016;2016:1-7.

30 Zijlmans MAC, Korpela K, Riksen-Walraven JM, et al. Maternal prenatal stress is associated with the infant intestinal microbiota. Psychoneuroendocrinology 2015;53:233-45

31 Zimmermann $\mathrm{P}_{1}$ Messina N, Mohn WW, et al. Association between the intestinal microbiota and allergic sensitization, eczema, and asthma: a systematic review. J Allergy Clin Immunol 2019;143:467-85.

32 Nilsen RM, Vollset SE, Gjessing HK, et al. Self-selection and bias in a large prospective pregnancy cohort in Norway. Paediatr Perinat Epidemiol 2009;23:597-608.

33 Asher MI, Montefort S, Björkstén B, et al. Worldwide time trends in the prevalence of symptoms of asthma, allergic rhinoconjunctivitis, and eczema in childhood: ISAAC phases one and three repeat multicountry cross-sectional surveys. Lancet 2006;368:733-43.

34 Nivel Zorgregistraties eerste lijn. Available: https://www.nivel.nl/nl/nivelzorgregistraties-eerste-lijn/nivel-zorgregistraties-eerste-lijn [Accessed 01-06-2020]. 